\title{
Changes to the varicella and pertussis immunisation schedule in Germany 2009: Background, rationale and implementation
}

M Wiese-Posselt (Wiese-PosseltM@rki.de) ${ }^{1,2}$, W Hellenbrand $^{1,2}$

1. Immunisation Unit, Department for Infectious Disease Epidemiology, Robert Koch Institute, Berlin, Germany

2. Both authors contributed equally to this article.

In July 2009, the German Standing Committee on Vaccination (STIKO) modified its recommendations for varicella and pertussis vaccination, based on newly available data on disease epidemiology, vaccine effectiveness (VE) and safety, and an evaluation of the feasibility of the recommended immunisation strategy. The recommendation for varicella vaccine now includes a routine two-dose schedule with the administration of the first dose at the age of 11 to 14 months and the second dose at the age of 15 to 23 months, with a minimum interval of four weeks between these doses. Furthermore, STIKO recommended adding a one-time pertussis booster to the adult vaccination schedule to expand the cocoon strategy in place since 2004. The recommendation of a booster vaccination with an acellular pertussis vaccine every 10 years for persons employed in the care of pre-school children and for healthcare personnel in paediatric, gynaecologic and obstetric health facilities was extended to persons employed in schools and in other institutions caring for older children, and to all healthcare personnel. These recommendations were based on available epidemiological data showing an increase in incidence from 7-10 cases per 100,000 inhabitants in 2002-2004 to over 30 by 2007 . Moreover, the high burden of pertussis in infants at 94 hospitalised cases per 100,000 infants in 2007 suggested that the previous cocoon strategy was insufficient.

\section{Introduction}

The German Standing Committee on Vaccination (STIKO) is a board of honorary experts in the fields of vaccinology, infectious disease epidemiology, microbiology and virology, paediatrics, evidence-based medicine and others. STIKO members are appointed by the Federal Ministry of Health every three years. STIKO reviews its recommendations for vaccination on an annual basis. Its decision-making process is based on published data on disease epidemiology, vaccine effectiveness (VE), and safety, and on the feasibility of the recommended immunisation strategy. STIKO is not responsible for a health economic assessment of the effect of vaccination. In this paper we communicate the major modifications to the immunisation schedule in Germany implemented in July 2009 and we detail the scientific rationale for the changes [1]. The main changes were the addition of a second dose of varicella vaccine to the routine childhood vaccination schedule and the recommendation of a single pertussis booster for adults.

So far, Germany is the only country in Europe to have introduced a nationwide recommendation for routine childhood varicella vaccination. Since the introduction of, initially, a one-dose varicella vaccination in children, a significant reduction of varicella cases in children was observed in a nationwide sentinel surveillance system, as recently published by Siedler et al. [2]. This positive experience with varicella vaccination in Germany and the deliberations of the STIKO on the introduction of a two-dose varicella vaccination schedule might be helpful for other countries in Europe in their decision making process.

Second dose of varicella vaccine in children In 2004, STIKO recommended routine varicella vaccination for all children older than 11 months with the aim of reducing varicella associated morbidity and the reduction of the burden of disease [3]. A single dose of monovalent varicella vaccine was recommended at 11 to 14 months of age and at that time, two licensed vaccines were available for this purpose in Germany, Varilrix and Varivax. In 2006, the measles-mumpsrubella-varicella (MMRV) vaccine (Priorix-Tetra) was licensed for administration in two doses at least four weeks apart [4]. In 2008, the licensed immunisation schedule of both monovalent varicella vaccines changed to a two-dose scheme for all children $[5,6]$.

Based on epidemiological data from Germany and the United States (US) as well as newly available data on varicella zoster virus (VZV) vaccine immunogenicity and effectiveness after one and two doses of VZV-containing vaccines, STIKO decided to follow 
the change of vaccine licensures and recommended a routine two-dose vaccination strategy in children. The aim of this recommendation is to reduce the number of varicella outbreaks, the number of cases with varicella breakthrough illness (BI) and the transmission of VZV to susceptible persons with a higher risk for severe varicella disease such as pregnant women and immunocompromised patients.

\section{Epidemiological data from}

Germany and the US

Varicella is not notifiable in Germany. Therefore, a country-wide sentinel project on varicella and zoster epidemiology was established in Germany in April $2005[7,8]$. The constitution of the sentinel system and its first results covering the time frame April 2005 to March 2009 have been published recently [2]. Since sentinel surveillance cannot provide population-based data, varicella incidence and vaccination coverage cannot be calculated directly. However, from 2005 to 2009, physicians reporting to the sentinel diagnosed successively fewer children with varicella with a significantly decreasing trend over time, while they administered a rapidly increasing number of varicella vaccines for first doses and since 2008, second doses of VZVcontaining vaccine as well. A stable number of sentinel physicians reported around $60 \%$ fewer varicella cases in the 2008-9 season than in the 2005-6 season. Concurrently, a rising number of varicella $\mathrm{BI}$ was reported over the observation period $[2,8]$.

Available information on vaccine coverage for the federal state of Schleswig-Holstein shows that coverage in children under 24 months of age with one dose of VZV containing vaccine, increased from $11 \%$ in 2006 to $83 \%$ in 2008 [personal communication Hans-Martin Bader and Maik Ludwig].

In 2008 and 2009, the RKI investigated seven varicella outbreaks in day-care centres including 631 children with available varicella vaccination information [9]. The attack rate was $13 \%$ in vaccinated children and $48 \%$ in unvaccinated children, resulting in an overall VE of $71 \%$ [95\% confidence interval $(\mathrm{Cl}): 57-81$ ] in the multivariable analysis [9]. VE differed significantly by disease severity and number of doses administered. VE of one dose and two doses of VZV-containing vaccines was estimated to be $62 \%$ [95\% Cl: $43-75$ ] and $94 \%$ [95\% Cl: 75-98], respectively. Furthermore, results of outbreak investigations in Germany suggest that VE and the risk of varicella $\mathrm{BI}$ are not uniform for the different varicella vaccine products available in Germany [9].

The US varicella vaccination programme implemented in 1996 recommended one dose of monovalent varicella vaccine at the age of 12 to 18 months and achieved over $90 \%$ reduction in varicella morbidity in children between 1995 and 2005 [10,11]. Since varicella outbreaks continued to occur and an increasing number of cases with varicella $\mathrm{BI}$ was observed in active surveillance areas, the US Advisory Committee on Immunization Practices (ACIP) recommended a second dose of varicella vaccine for all children aged four to six years in 2007 [12]. However, when applying these data to the German situation, it must be kept in mind that there is only one vaccine (Varivax) available in the US and a high vaccination coverage over $90 \%$ in children under three years old has been achieved for a prolonged period of time [11].

\section{Effectiveness, immunogenicity, and} safety of VZV-containing vaccines VE after one dose Varivax was estimated at $84.5 \%$ (range: 44-100) in a recent review [13]. Estimates from three epidemiological studies on the VE of one dose of Varilrix range from 20 to $92 \%$ [14-16]. So far no data on VE after two doses of Priorix-Tetra have been published.

In 2008, the licensed immunisation schedule of both monovalent varicella vaccines changed to a two-dose scheme for all children $[5,6]$. The regulatory authorities based this change of licensure on immunogenicity data after one and two doses of VZV containing vaccines and on a large epidemiological study by Kuter et al. conducted in the US in the 1990s using Varivax, although at a higher potency than available licensed vaccines [17-19]. In this study the risk of developing varicella 42 days after vaccination was 3.3-fold lower in children who received two doses of Varivax compared with those who received only one dose ( $p<0.001)$. The estimated vaccine efficacy for the 10-year observation period for one or two doses Varivax was $94.4 \%$ and $98.3 \%$, respectively ( $p<0.001)$. This efficacy estimate was based on comparison with historical data of annual varicella incidences in unvaccinated susceptible children. In a randomised controlled trial in 10-21 month-old VZV seronegative children an over 20 -fold higher geometric mean titre (GMT) of VZV antibodies was observed in children with two doses of PriorixTetra compared with children vaccinated with only one dose of a monovalent varicella vaccine [17]. However, the study by Kuter et al. showed that GMTs decreased to similar levels within several years regardless of the number of doses (one or two) applied [19]. Therefore, the reliability of VZV antibody levels as a correlate of protection after vaccination remains unclear.

A good safety profile of VZV-containing vaccines available in Germany has been reported [4-6]. However, for the MMRV vaccine ProQuad, which is only available in the US, febrile seizures were observed more frequently post-vaccination than after simultaneous administration of MMR and monovalent varicella vaccines in children under two years of age. [20].

\section{Feasibility of routine two-dose}

varicella vaccination in children

The first dose of VZV-containing vaccine is recommended at the age of 11 to 14 months and the second dose at the age of 15 to 24 months. Thus, varicella vaccination can be administered simultaneously with the 
measles-mumps-rubella (MMR) vaccination using a monovalent varicella vaccine plus an MMR vaccine or using the licensed MMRV vaccine. However, varicella vaccination can be offered to any child aged 11 months or older. A second dose is also recommended for all children who thus far received only one dose of VZVcontaining vaccine.

\section{Pertussis vaccination of adults}

In July 2009, STIKO recommended adding a single pertussis booster to the adult vaccination schedule against pertussis, in order to expand the cocoon strategy which recommended pertussis vaccination for all adults with close contact to infants since 2004. The 2004 recommendations also included a booster vaccination with an acellular pertussis (ap) vaccine every 10 years for persons employed in the care of pre-school children and for healthcare personnel in paediatric, gynaecologic and obstetric health facilities. These were expanded to include persons employed in schools and in other institutions caring for older children, as well as all healthcare personnel. The new recommendations were based on careful analysis of available epidemiological data on the disease burden in adults as well as data on immunogenicity, effectiveness and safety of available vaccines. The aim of the updated recommendations is to decrease pertussis-related morbidity in adults as well as in non-immune, unprotected contacts, particularly infants [21;22].

Epidemiological data from Germany for adults Pertussis is a notifiable disease in five of the 16 German federal states. Despite increasing vaccination coverage among children at school entry to over $93 \%$, the incidence of pertussis in adults in the states with statutory surveillance increased from 7-10 cases per 100,000 inhabitants in 2002-2004 to a maximum of 32 in 2007 , decreasing only slightly to 30 in 2008 [22;23]. The average age of notified cases increased from 15 years in 1995 to 42 years in 2008 , with a concomitant increase in the proportion of cases older than 19 years (adult cases) from 20 to $75 \%$. Sentinel surveillance performed from 2000-2004 [24] estimated a similar annual incidence in an east and a west German city, 169 and 160 pertussis cases per 100,000 adult inhabitants, respectively, translating to a nationwide estimated incidence of 160 . However, pertussis incidence increased according to statutory surveillance in the years following conclusion of this sentinel study. These figures thus suggested a marked under-ascertainment in the statutory surveillance system. In addition, the burden of pertussis in infants remains high with an incidence of 94 hospitalised cases per 100,000 infants in 2007, implying that the cocoon strategy implemented in 2004 was insufficient.

The majority of pertussis outbreaks notified from 2002-2007 in the five German federal states with statutory pertussis surveillance involved children older than nine years $(70 \%$ of cases in outbreaks with more than five cases), which was the rationale for expanding the recommendation for pertussis vaccination to persons employed in schools and other institutions involved in the care of older children. A Canadian study calculated that healthcare personnel had a 1.7-fold elevated risk for pertussis compared with the general population [25]. Numerous pertussis outbreaks among healthcare personnel involved in the care of adults have been published [26] and a modelling study in the United Kingdom estimated a high incidence of pertussis in hospitalised elderly patients [27].

Adults with pertussis often fail to present with the full-blown clinical picture, but nonetheless suffer significant morbidity. In the above mentioned surveillance study by Riffelmann et al. [24], the median duration of the primary symptom, cough, was 48 days (maximum: 72 weeks) and on average, patients consulted their general physician 5.4 times and $27 \%$ were referred to a specialist. Antibiotics were prescribed for $53 \%$ of patients and $13 \%$ received steroids. In various case series between $23 \%$ and $28 \%$ of all adult patients with pertussis suffered complications, including sinusitis, otitis media, incontinence, weight loss, rib fractures, syncope and pneumonia $[24 ; 25 ; 28 ; 29]$. Hospitalisation was reported in 1 to $3 \%$ of adult pertussis cases [25;29-31].

\section{Effectiveness, immunogenicity and}

safety of pertussis vaccines

Effectiveness of vaccination with an ap vaccine containing three pertussis antigens was demonstrated in adults in one randomised controlled trial, which was, however, based on the observation of only 10 pertussis cases in total: one case amongst 1,391 tetanus-diphtheria-acellular pertussis (Tdap)-vaccinated participants, and nine pertussis cases amongst 1,390 hepatitis A vaccinated participants [34]. Vaccine efficacy was estimated at $92 \%(95 \% \mathrm{Cl} 32-99)$. Numerous studies have demonstrated immunogenicity of pertussis vaccines in adults equivalent to that observed in studies in children in which clinical efficacy was shown [32]. These studies have also demonstrated that the safety and reactogenicity of ap vaccines is similar to that of $\mathrm{Td}$ (tetanus-diphtheria) vaccines.

\section{Cost-effectiveness}

Lee et al. [33] performed a cost-effectiveness analysis for vaccination of adults in Germany using conservative estimates of pertussis incidence and including direct costs as well as indirect costs due to loss of work by means of a Markov model. This suggested that one time vaccination of around $62 \%$ of adults would be cost-effective and possibly cost-saving, at a cost of 160 Euros per pertussis case prevented. Two other models tailored to the US situation came to similar conclusions [21;34;35].

\section{Feasibility of implementing a one- time adult pertussis booster} Implementation of an adult booster is considered feasible. Telephone surveys in Germany revealed a high 
acceptance of tetanus vaccination, with $75 \%$ of a population based sample of adults reporting having received a tetanus-containing vaccination in the past 10 years (unpublished data, RKI). However, it is essential that Tdap vaccines are made widely available for tetanus post-exposure immunisation in emergency settings. In addition, efforts are needed to improve vaccination coverage in adolescents, which remains low at $36 \%[36]$.

\section{Conclusions}

\section{Varicella vaccination}

Germany is currently the only country in Europe that includes varicella vaccination in the routine immunisation schedule for children. Results from sentinel surveillance show that the recommendation of routine varicella vaccination for all children older than 11 months in 2004 has had a positive effect on the overall burden of varicella in Germany [2]. Based on immunogenicity data after one and two doses VZV-containing vaccines (indirect evidence) and based on the findings of the study of Kuter et al., licensures of both monovalent varicella vaccines changed to a two-dose schedule [17-19]. With respect to recent epidemiological findings in Germany and from the US, where varicella vaccines are included in the routine immunisation programmes since $1996[2,11]$, STIKO decided to follow the change of licensure and recommended a second dose for all children in July 2009. As published VE estimates vary widely, and one VZV vaccine (ProQuad) has been associated with an increased risk of febrile seizures in children less than 2 years of age $[13-16,20]$, there is a need for additional post-marketing data on safety and effectiveness of VZV-containing vaccines. A populationbased VZV surveillance system should be established in Germany to evaluate the effect of routine varicella vaccination in children and to monitor varicella and herpes zoster incidences in all age groups.

\section{Pertussis vaccination}

As herd protection has been demonstrated after implementation of pertussis vaccination in children [37;38], it seems reasonable to assume that vaccination of adults can lead to reduction of disease in unprotected individuals, particularly infants. Furthermore, a modelling study has estimated that around $34 \%$ of all transmission of pertussis to infants occurs via less close contacts [39]. Both natural and vaccine-induced immunity is not lifelong [40], waning immunity in the face of decreased natural boosting in conjunction with increasing vaccination coverage in children [41] - is likely the most important factor leading to high disease burden in adults. The aim of the addition of a pertussis booster to the adult vaccination schedule is thus to reduce the overall pertussis disease burden. The implementation of the changes to the adult pertussis vaccination schedule will be evaluated in regular population-based telephone interviews soliciting data on vaccination status, and through analysis of routine surveillance data available from the former East German states, hospital discharge statistics and the established laboratory surveillance of molecular genetic characteristics of $B$. Pertussis.

\section{References}

1. Robert Koch-Institut. Empfehlungen der Ständigen Impfkommission (STIKO) am Robert Koch-Institut. [Recommendation of the Standing Vaccination Commission (STIKO) at the Robert Koch-Institute]. Epid Bull 2009;30. [German]. Available from: http://www.rki.de/cln_16o/ nn_195850/DE/Content/Infekt/Impfen/Impfempfehlungen/ Imp̄empfehlungen__node.html?_nnn=true

2. Siedler A, Arndt U. Impact of the routine varicella vaccination programme on varicella epidemiology in Germany. Euro Surveill. 2010;15(13). pii=19530. Available from: http://www. eurosurveillance.org/ViewArticle.aspx?Articleld=19530

3. Robert Koch-Institut. Empfehlungen der Ständigen Impfkommission am Robert Koch-Institut. [Recommendation of the Standing Vaccination Commission (STIKO) at the Robert Koch-Institute]. Epid Bull 2004;30:235-50. [German].

4. Glaxo Smith Kline: Product information of Priorix-Tetra $®$. October 2007.

5. Sanofi Pasteur MSD: Product information of Varivax $®$. August 2008.

6. Glaxo Smith Kline: Product information of Varilrix ${ }^{\circledR}$. August 2008.

7. Robert Koch-Institut. Zur Sentinel-Surveillance der Varizellen in Deutschland. [Regarding the sentinal surveillance of varicella in Germany]. Epid Bull. 2008;8;61-4. Available from: http:// www.rki.de/cln_16o/nn_969736/DE/Content/Infekt/EpidBull/ Archiv/2008/08/Tabelle.html? nnn=true [German]

8. Robert Koch-Institut. [Gemeinsamer Varizellen-Workshop von AGMV und BaVariPro]. Epid Bull. 2010;8:69-75. [German]

9. Spackova M, Wiese-Posselt M, Dehnert M, Matysiak-Klose $D$, Heininger U, Siedler A. Comparative varicella vaccine effectiveness during outbreaks in day-care centres. Vaccine. 2010;28(3):686-91.

10. Centers for Disease Control and Prevention. Prevention of varicella: recommendations of the Advisory Committee on Immunization Practices (ACIP). MMWR Recomm Rep. 1996;45(RR-11). Available from: www.cdc.gov/mmwr/preview/ mmwrhtml/o0042990.htm

11. Guris D, Jumaan AO, Mascola L, Watson BM, Zhang JX, Chaves SS, et al. Changing varicella epidemiology in active surveillance sites -- United States, 1995-2005. J Infect Dis. 2008;197(Suppl 2):S71-5.

12. Centers for Disease Control: Prevention of varicella: recommendations of the Advisory Committee on Immunization Practices (ACIP). MMWR Recomm Rep. 2007;56(RRo4);1-40.

13. Seward JF, Marin M, Vázquez M: Varicella vaccine effectiveness in the US vaccination program: a review. J Infect Dis. 2008;197(Suppl 2):S82-9.

14. Miron D, Lavi I, Kitov R, Hendler A. Vaccine effectiveness and severity of varicella among previously vaccinated children during outbreaks in day-care centers with low vaccination coverage. Pediatr Infect Dis J. 2005;24(3):233-6.

15. Sheffer R, Segal D, Rahamani S, Dalal I, Linhart Y, Stein M, et al. Effectiveness of the $0 \mathrm{ka} / \mathrm{GSK}$ attenuated varicella vaccine for the prevention of chickenpox in clinical practice in Israel. Pediatr Infect Dis J. 2005;24(5):434-7.

16. Passwell JH, Hemo B, Levi Y, Ramon R, Friedman N, Lerner-Geva L. Use of a computerized database to study the effectiveness of an attenuated varicella vaccine. Pediatr Infect Dis J. 2004;23(3):221-6.

17. Schuster V, Otto W, Maurer L, Tcherepnine P, Pfletschinger U, Kindler K, et al. Immunogenicity and safety assessments afte one and two doses of a refrigerator-stable tetravalent measlesmumps-rubella-varicella vaccine in healthy children during the second year of life. Pediatr Infect Dis J. 2008;27(8):724-30.

18. Vesikari T, Baer M, Willems P. Immunogenicity and safety of a second dose of measles-mumps-rubella-varicella vaccine in healthy children aged 5 to 6 years. Pediatr Infect Dis J. 2007;26(2):153-8

19. Kuter B, Matthews H, Shinfield H, Black S, Dennehy P, Watson $B$, et al. Ten year follow-up of healthy children who received one or two injections of varicella vaccine. Pediatr Infect Dis I. 2004;23:132-7.

20. Centers for Disease Control and Prevention: Advisory Committee on Immunization Practices (ACIP): ACIP Presentation Slides: October 2008 Meeting: MMRV vaccine safety. 
21. Wendelboe AM, Njamkepo E, Bourillon A, Floret DD, Gaudelus J, Gerber M et al. Transmission of Bordetella pertussis to young infants. Pediatr Infect Dis J. 2007;26(4):293-9.

22. Ständige Impfkommission am Robert Koch-Institut. Mitteilung der Ständigen Impfkommission (STIKO) am Robert KochInstitut: Zusätzliche Pertussis-Impfung im Erwachsenenalter als Tdap-Kombinationsimpfung bei der nächsten fälligen TdImpfung - Empfehlung und Begründung. [Communication from the Standing Vaccination Commission (STIKO) at the Robert Koch-Institute]. Epid Bull 2009;31:299-311. [German].

23. Hellenbrand W, Beier D, Jensen E, Littmann M, Meyer C, Oppermann $\mathrm{H}$ et al. The epidemiology of pertussis in Germany: past and present. BMC Infect Dis. 2009;9(22). Available from: http://www.biomedcentral.com/1471-2334/9/22.

24. Riffelmann M, Littmann M, Hülße C, O’Brien J, Wirsing von Konig CH, KRESH-Studienärzte. Pertussis bei Erwachsenen: Häufigkeit, Symptome und Kosten. [Pertussis: incidence, symptoms and costs]. Dtsch Med Wochenschr. 2006;131:282934. [German].

25. De Serres G, Shadmani R, Duval B, Boulianne N, Déry P, Douville Fradet $M$ et al. Morbidity of pertussis in adolescents and adults. J Infect Dis. 2000;182(1):174-9.

26. Ständige Impfkommission am Bundesgesundheitsamt. Mitteilung der Ständigen Impfkommission (STIKO) am Robert Koch-Institut: Erweiterung der beruflichen Indikationen für eine Pertussis-Impfung. [Communication from the Standing Vaccination Commission (STIKO) at the Robert Koch-Institute] Epid Bull 2009;31:311-3. [German].

27. Müller-Pebody B, Crowcroft NS, Zambon MC, Edmunds WJ. Modelling hospital admissions for lower respiratory tract infections in the elderly in England. Epidmiol Infect. 2006;134(6):1150-7.

28. Lee G, Lett S, Schauer S, LeBaron C, Murphy T, Rusinak D et al. Societal costs and morbidity of pertussis in adolescents and adults. Clin Infect Dis 2004;39(11):1572-80.

29. Postels-Multani S, Wirsing von Koenig CH, Schmitt HJ, Bock HL, Bogaerts H. Symptoms and complications of pertussis in adults. Infection. 1995;23(3):139-42.

30. Kretsinger K, Broder K, Cortese MM, Joyce PM, Ortega-Sanchez IR, Lee GM et al. Preventing tetanus, diphtheria, and pertussis among adults: use of tetanus toxoid, reduced diphtheria toxoid and acellular pertussis vaccine recommendations of the Advisory Committee on Immunization Practices (ACIP) and recommendation of ACIP, supported by the Healthcare Infection Control Practices Advisory Committee (HICPAC), for use of Tdap among health-care personnel. MMWR Recomm Rep. 2006;55(RR-17):1-37.

31. Trollfors B, Rabo E. Whooping cough in adults. Br Med J. 1981;283:696-7.

32. Ständige Impfkommission am Robert Koch-Institut. Appendix to: Mitteilung der Ständigen Impfkommission (STIKO) am Robert Koch-Institut: Zusätzliche Pertussis-Impfung im Erwachsenenalter als Tdap-Kombinationsimpfung bei der nächsten fälligen Td-Impfung - Empfehlung und Begründung. [Standing Vaccination Commission (STIKO) at the Robert KochInstitute]. Epid Bull 2009;31:299-311. [German].

33. Lee GM, Riffelmann M, Wirsing von Konig CH. Costeffectiveness of adult pertussis vaccination in Germany. Vaccine. 2008;26(29-30):3673-9.

34. Lee GM, Murphy TV, Lett S, Cortese MM, Kretsinger K, Schauer $S$ et al. Cost effectiveness of pertussis vaccination in adults. Am J Prev Med. 2007;32(3):186-93.

35. Purdy KW, Hay JW, Botteman MF, Ward JI. Evaluation of strategies for use of acellular pertussis vaccine in adolescents and adults: a cost-benefit analysis. Clin Infect Dis 2004;39:20-8.

36. Poethko-Müller C, Kuhnert R, Schlaud M. Vaccination coverage and predictors for vaccination level. Results of the German Health Interview and Examination Survey for Children and Adolescents (KiGGS). Bundesgesundheitsblatt Gesundheitsforschung Gesundheitsschutz. 2007;50(5-6):851-62.

37. Rohani P, Earn DJ, Grenfell BT. Impact of immunisation on pertussis transmission in England and Wales. Lancet 2000;355(9200):285-6.

38. Taranger J, Trollfors B, Bergfors E, Knutsson N, Sundh V, Lagergard T et al. Mass vaccination of children with pertussis toxoid -- decreased incidence in both vaccinated and nonvaccinated persons. Clin Infect Dis 2001;33(7):1004-10.

39. Wendelboe A, Hudgens M, Poole C, Van Rie A. Estimating the role of casual contact from the community in transmission of Bordetella pertussis to young infants. Emerg Themes Epidemiol. 2007;4(1):15.
40. Wendelboe AM, Van Rie A, Salmaso S, Englund JA. Duration of immunity against pertussis after natural infection or vaccination. Pediatr Infect Dis J. 2005;24(5 Suppl):S58-61.

41. Aguas R, Goncalves G, Gomes MG. Pertussis: increasing disease as a consequence of reducing transmission. Lancet Infect Dis. 2006;6(2):112-7. 